\title{
Factors associated with a clinically relevant reduction in menopausal symptoms of a standardized acupuncture approach for women with bothersome menopausal symptoms
}

Frans Boch Waldorff ${ }^{1,2^{*}}$ (D), Christine Winther Bang ${ }^{1}$, Volkert Siersma ${ }^{1}$, John Brodersen ${ }^{1,3}$ and Kamma Sundgaard Lund ${ }^{1}$

\begin{abstract}
Background: Little is known about factors associated with a clinically relevant reduction in menopausal symptoms through a brief acupuncture approach for women with moderate-to-severe menopausal symptoms.

Methods: Post hoc analysis of a randomized controlled trial where participants were allocated to early versus late standardized acupuncture. Both the early group and the late group are included in this study. The late group got an identical intervention parallel staged by 6 weeks. By means of the relative importance, the effect was evaluated for both early versus late women with a 6-week follow-up. We included four symptom subscales from the validated MenoScores Questionnaire: hot flushes, day and night sweats, general sweating, menopausal-specific sleeping problems, as well as an overall score, which is the sum of the four outcomes in the analysis.

Results: 67 women with moderate to severe menopausal symptoms were included of whom 52 (77.6\%) experienced a clinically relevant reduction in any of the four surveyed symptom subscales or overall score. 48 (71.6\%) women experienced a clinically relevant reduction in any of the vasomotor symptom subscales: hot flushes, day and night sweats, general sweating.

Women with vocational education were most likely to experience improvement compared to women with higher education. Beyond education, other factors of some importance for a clinically relevant reduction were no alcohol consumption, two or more births and urinary incontinence.
\end{abstract}

Conclusions: Level of education was the most consistent factor associated with improvement. Beyond education, other factors of some importance were no alcohol consumption, two or more births and urinary incontinence.

(Continued on next page)

\footnotetext{
* Correspondence: fransw@sund.ku.dk

${ }^{1}$ The Research Unit for General Practice and Section of General Practice, Department of Public Health, University of Copenhagen, Copenhagen, Denmark

${ }^{2}$ Department of Public Health, University of Southern Denmark, Research Unit of General Practice, Odense, Denmark

Full list of author information is available at the end of the article
}

(c) The Author(s). 2021 Open Access This article is licensed under a Creative Commons Attribution 4.0 International License, which permits use, sharing, adaptation, distribution and reproduction in any medium or format, as long as you give appropriate credit to the original author(s) and the source, provide a link to the Creative Commons licence, and indicate if changes were made. The images or other third party material in this article are included in the article's Creative Commons. licence, unless indicated otherwise in a credit line to the material. If material is not included in the article's Creative Commons licence and your intended use is not permitted by statutory regulation or exceeds the permitted use, you will need to obtain permission directly from the copyright holder. To view a copy of this licence, visit http://creativecommons.org/licenses/by/4.0/. The Creative Commons Public Domain Dedication waiver (http://creativecommons.org/publicdomain/zero/1.0/) applies to the data made available in this article, unless otherwise stated in a credit line to the data. 
(Continued from previous page)

Trial registration: This study was registered in ClinicalTrials.gov at April 21, 2016. The registration number is NCT02 746497.

Keywords: Acupuncture, Clinical trials, Primary care, Menopause

\section{Background}

Menopausal symptoms are common and may affect quality of life, health status, and use of health services [1-3]. Most women experience menopause in their early 50s [4] and the symptoms last for $4-5$ years on average [4-7]. The key symptom of menopause is hot flushes, which affects around $75 \%$ of menopausal women $[5,6$, $8]$ and up to $10-20 \%$ report this symptom as very bothersome [5]. Other frequent menopausal symptoms are sweating and sleeping problems [9].

Previously, we have reported that a standardized and brief acupuncture treatment produced a fast and clinically relevant reduction among women with moderate-tosevere hot flushes, day and night sweating, general sweating as well as sleeping problems [10] and that overall effect on menopause-relevant outcomes was sustained up to 21 weeks after treatment stopped [11]. However, the literature on determinants for the effect of acupuncture is sparse [12], which is important to provide guidance to both the women and the acupuncturist.

Therefore, the objective of this study was to investigate which factors are important for a clinically relevant reduction in menopausal symptoms in a standardized brief acupuncture approach for women with moderate-tosevere hot flushes.

\section{Methods}

\section{Design and intervention}

The study used data from a randomized trial evaluating the effectiveness of a standardized acupuncture intervention for women with moderate to severe menopausal symptoms [10]. We included healthy women aged $40-$ 65 years experiencing moderate to severe menopausal symptoms evaluated by the hot flushes (HF) subscale from the MenoScores Questionnaire (MSQ) [9]. Women were excluded if they had undergone hysterectomy and/ or bilateral oophorectomy, if they used other treatments for menopausal symptoms or treatments that might affect menopausal symptoms or if they had received acupuncture treatment within the past six months before enrolment. All participants were provided with oral and written information about the study and participation was voluntary, the participant could withdraw their consent at any time.

Women were randomly allocated to an early intervention $(n=36)$ and late intervention $(n=34)$ with a cross over at week 6 . The intervention in both groups were identical but parallel staggered by 6 weeks. Women from both the early and late group are included in this study and we included allocation status as a confounder in the analysis.

The intervention consisted of one treatment with a needle retention time of $10 \mathrm{~min}$ every week for five week. The acupuncture was Western medical acupuncture (WMA) [13] and the predefined acupuncture points were: CV-3, CV-4, LR-8, SP-6, SP-9 (LR-8, SP-6 and SP9 bilaterally). The acupuncturists were all GPs with on average $153 \mathrm{~h}$ of acupuncture education (range 80-300) and had practiced acupuncture for 14 years (range 4-38 years). All but one were educated in acupuncture by the Danish Society for Evidence-based Acupuncture (DSEA) or the Danish Medical Acupuncture Society. Further details on the intervention are available in our previously published paper [14].

\section{Assessments}

The importance of a selection of factors was evaluated on four symptom subscales from the MSQ [9] - three vasomotor symptom (VMS) subscales: HF, day and night sweats (DNS), general sweating (GS), and menopausal-specific sleeping problems (MSSP) - as well as an overall score which is the sum of the four outcomes. The MSQ is a validated condition-specific patient-reported outcome measure consisting of 11 subscales of which the above four are most directly HF connected to menopause [9]. The scores of each of the symptom scales were related to a global item inquiring into whether the woman was bothered by menopausal symptoms. The difference in mean symptom score from 'a lot' on the global item to 'quite a bit' on the global item was considered a clinically relevant reduction. For each of the symptom scales this corresponded to a slightly different point reduction, which translated to a reduction with at least two points since the symptom scores are integer. Similarly, the clinically relevant reduction in the overall score was found to be nearby six points. Clinically relevant reductions of 2 points respectively 6 points were also found when the difference in mean symptom scores from "quite a bit" to "a bit" were considered.

All participants were asked to complete the MSQ before during and after the acupuncture treatment.

\section{Factors investigated for their relative importance}

The factors investigated for their importance in obtaining a clinically relevant reduction in menopause-specific 
symptoms were all the variables that were collected from the baseline questionnaire completed by all women at inclusion into the ACOM study. This selection of factors was:

- Age, Chronic conditions, Body Mass Index

- Menopause-related factors: Menstruation last year, Duration of hot flushes, Number of births, Incontinence.

- Social factors: Level of education: Vocational, short and medium cycle higher education, long cycle higher education, and other (not specified education or don't know). Employment: employed, unemployed, Household status: living alone, living with others, Smoking: yes, no, Alcohol use: no alcohol, $\leq 14$ units per week, $>14$ units per week, Physical activity: no, 1-3 times per week, $\geq 4$ times per week, Previous experience with acupuncture: no, yes.

- Further, we included allocation status in the analysis.

\section{Statistical analysis}

The relative importance of the above factors for each of the four menopause-specific subscales and for the overall score was assessed in a dominance analysis [15]. A dominance analysis attempts to distribute the explained variance of the full multivariable model into the parts attributable to the individual factors. In the present analyses, the model was a multivariable logistic regression model and its fit was assessed by McFadden's pseudo $R^{2}$. The relative importance of each factor was computed as the mean increase in the pseudo $R^{2}$ value obtained by adding the factor to each possible model containing the remaining variables. The relative importances were then normalized such that they add up to 1 . The implemented definition of relative importance explicitly accounts for correlation between factors.

To characterize the effect of the most important factors a multivariable logistic regression with the top three important factors for each of the outcomes was performed.

\section{Results}

Mean age of the women were 54.8 years and on average, they experienced hot flushes for 4.1 years. Baseline data stratified by experienced clinical reduction in the four symptom scales and overall composite scale is presented in Table 1.

$52(77.6 \%)$ experienced a clinically relevant reduction in any of the four symptom scales, 48 (71.6\%) in any of the VMS subscales, and $32(47.8 \%)$ in the overall composite score, respectively.

Women with vocational education were most likely to experience improvement compared to women with longer education. Beyond level of education, other factors of some importance for clinically relevant reduction were no alcohol consumption, two or more births and urinary incontinence (Table 2).

\section{Discussion}

Women with vocational education were most inclined to experience an improvement in menopausal symptoms while women with higher education to a lesser degree experienced improvement. Beyond level of education, other factors of some importance for clinically relevant reduction were no alcohol consumption, two or more births and urinary incontinence.

Around 12\% of Danish women between 45 and 64 years stated that they had used acupuncture within the last year [16]. Women with longer education were less likely to use acupuncture. This may be due to a more critical perception towards acupuncture or a lower expectation towards the efficacy of acupuncture. Perception of and expectations has been identified as central to the perceived efficacy of acupuncture analgesia [17] and we hypothesize that the same mechanisms may explain our findings. However, we do find that current use of alternative medication actually decrease the effect acupuncture in the sleeping dimension, but not in any other dimensions.

A moderate consumption of alcohol has been associated with delayed menopausal onset [18], which may be due to an estrogenic effect caused by alcohol [19]. The mechanisms behind our findings were difficult to explain based on the design of our study. In order to study these mechanisms we suggest conducting research to explore perceptions and experiences of acupuncture for women with moderate to severe menopausal symptoms.

Previous studies on the effect of acupuncture treatment for menopausal symptoms have typically focused on VMS and reported a 30-60\% reduction in VMS frequency among women receiving treatment [20-25]. This is in line with our findings on the composite score (48\%). However, these studies report aggregated data and consequently do not identify factors associated with a perceived clinically relevant effect. Further, the outcomes used are not obtained from validated scales and the interventions are characterized by individualized acupuncture modalities.

Only one study has surveyed the probability for a reduction in VMS over 8 weeks: A small group (11\%) experienced an $80 \%$ reduction in VMS, 59\% reported a $40 \%$ reduction and $41 \%$ did not experience a reduction in VMS frequency at 8 weeks [12]. In our study 48 (72\%) reported a clinically relevant reduction in any of the three VMS subscales. It is difficult directly to compare our results due to our use of a validated outcome and our definition of a clinically relevant outcome [9]. 
Table 1 Baseline data stratified by experienced clinical reduction in the four symptom scales and overall composite scale

\begin{tabular}{|c|c|c|c|c|c|c|c|c|c|c|c|}
\hline & \multirow[t]{2}{*}{ Total } & \multicolumn{2}{|c|}{$\begin{array}{l}\text { Hot flushes } \\
\text { Clinical reduction }\end{array}$} & \multicolumn{2}{|c|}{$\begin{array}{l}\text { Day-and-night } \\
\text { sweats } \\
\text { Clinical reduction }\end{array}$} & \multicolumn{2}{|c|}{$\begin{array}{l}\text { General sweating } \\
\text { Clinical reduction }\end{array}$} & \multicolumn{2}{|c|}{$\begin{array}{l}\text { Menopausal- } \\
\text { specific sleeping } \\
\text { problems } \\
\text { Clinical reduction }\end{array}$} & \multicolumn{2}{|c|}{$\begin{array}{l}\text { Overall } \\
\text { Clinical reduction }\end{array}$} \\
\hline & & Yes & No & Yes & No & Yes & No & Yes & No & Yes & No \\
\hline Total, n & 67 & 43 & 24 & 33 & 34 & 19 & 48 & 40 & 27 & 32 & 35 \\
\hline Age, mean (sd) & $\begin{array}{l}54.81 \\
(4.53)\end{array}$ & $\begin{array}{l}54.86 \\
(4.03)\end{array}$ & $\begin{array}{l}54.71 \\
(5.41)\end{array}$ & $\begin{array}{l}55.06 \\
(4.17)\end{array}$ & $\begin{array}{l}54.56 \\
(4.91)\end{array}$ & $\begin{array}{l}54.63 \\
(4.54)\end{array}$ & $\begin{array}{l}54.88 \\
(4.58)\end{array}$ & $\begin{array}{l}54.65 \\
(4.24)\end{array}$ & $\begin{array}{l}55.04 \\
(5.01)\end{array}$ & $\begin{array}{l}55.03 \\
(4.46)\end{array}$ & $\begin{array}{l}54.6 \\
(4.65)\end{array}$ \\
\hline \multicolumn{12}{|l|}{ Employment, n (\%) } \\
\hline Employed & $\begin{array}{l}60 \\
(89.55)\end{array}$ & $39(90.7)$ & $21(87.5)$ & $\begin{array}{l}30 \\
(90.91)\end{array}$ & $\begin{array}{l}30 \\
(88.24)\end{array}$ & $\begin{array}{l}17 \\
(89.47)\end{array}$ & $\begin{array}{l}43 \\
(89.58)\end{array}$ & $36(90)$ & $\begin{array}{l}24 \\
(88.89)\end{array}$ & $28(87.5)$ & $\begin{array}{l}32 \\
(91.43)\end{array}$ \\
\hline Unemployed & $7(10.45)$ & $4(9.3)$ & $3(12.5)$ & $3(9.09)$ & $4(11.76)$ & $2(10.53)$ & $5(10.42)$ & $4(10)$ & $3(11.11)$ & $4(12.5)$ & $3(8.57)$ \\
\hline \multicolumn{12}{|l|}{ Education, n (\%) } \\
\hline Vocational & $\begin{array}{l}16 \\
(23.88)\end{array}$ & $\begin{array}{l}16 \\
(37.21)\end{array}$ & $0(0)$ & $\begin{array}{l}11 \\
(33.33)\end{array}$ & $5(14.71)$ & $6(31.58)$ & $\begin{array}{l}10 \\
(20.83)\end{array}$ & $14(35)$ & $2(7.41)$ & $\begin{array}{l}13 \\
(40.63)\end{array}$ & $3(8.57)$ \\
\hline $\begin{array}{l}\text { Short or medium cycle higher } \\
\text { education }\end{array}$ & $7(10.45)$ & $3(6.98)$ & $4(16.67)$ & $3(9.09)$ & $4(11.76)$ & $3(15.79)$ & $4(8.33)$ & $3(7.5)$ & $4(14.81)$ & $3(9.38)$ & $\begin{array}{l}4 \\
(11.43)\end{array}$ \\
\hline Long cycle higher education & $\begin{array}{l}35 \\
(52.24)\end{array}$ & $\begin{array}{l}19 \\
(44.19)\end{array}$ & $\begin{array}{l}16 \\
(66.67)\end{array}$ & $\begin{array}{l}14 \\
(42.42)\end{array}$ & $\begin{array}{l}21 \\
(61.76)\end{array}$ & $8(42.11)$ & $\begin{array}{l}27 \\
(56.25)\end{array}$ & $17(42.5)$ & $\begin{array}{l}18 \\
(66.67)\end{array}$ & $\begin{array}{l}11 \\
(43.38)\end{array}$ & $\begin{array}{l}24 \\
(68.57)\end{array}$ \\
\hline Other & $9(13.43)$ & $5(11.63)$ & $4(16.67)$ & $5(15.15)$ & $4(11.76)$ & $2(10.53)$ & $7(14.58)$ & $6(15)$ & $3(11.11)$ & $5(15.63)$ & $\begin{array}{l}4 \\
(11.43)\end{array}$ \\
\hline \multicolumn{12}{|l|}{ Household } \\
\hline Living alone & $5(7.46)$ & $4(9.3)$ & $1(4.17)$ & $2(6.06)$ & $3(8.82)$ & $1(5.26)$ & $4(8.33)$ & $5(12.5)$ & 0 & $3(9.38)$ & $2(5.71)$ \\
\hline Living with others & $\begin{array}{l}62 \\
(92.54)\end{array}$ & $39(90.7)$ & $\begin{array}{l}23 \\
(95.83)\end{array}$ & $\begin{array}{l}31 \\
(93.94)\end{array}$ & $\begin{array}{l}31 \\
(91.18)\end{array}$ & $\begin{array}{l}18 \\
(94.74)\end{array}$ & $\begin{array}{l}44 \\
(91.67)\end{array}$ & $35(87.5)$ & $27(100)$ & $\begin{array}{l}29 \\
(90.63)\end{array}$ & $\begin{array}{l}33 \\
(94.29)\end{array}$ \\
\hline \multicolumn{12}{|l|}{ Physical activity, n (\%) } \\
\hline No physical activity & $\begin{array}{l}10 \\
(14.93)\end{array}$ & $4(9.3)$ & $6(25)$ & $4(12.12)$ & $6(17.65)$ & $1(5.26)$ & 9 (18.75) & $5(12.5)$ & $5(18.52)$ & $4(12.5)$ & $\begin{array}{l}6 \\
(17.14)\end{array}$ \\
\hline 1-3 times per week & $40(59.7)$ & $\begin{array}{l}30 \\
(69.77)\end{array}$ & $\begin{array}{l}10 \\
(41.67)\end{array}$ & $\begin{array}{l}20 \\
(60.61)\end{array}$ & $\begin{array}{l}20 \\
(58.82)\end{array}$ & $\begin{array}{l}12 \\
(63.16)\end{array}$ & $\begin{array}{l}28 \\
(58.33)\end{array}$ & $26(65)$ & $\begin{array}{l}14 \\
(51.85)\end{array}$ & $20(62.5)$ & $\begin{array}{l}20 \\
(57.14)\end{array}$ \\
\hline$\geq 4$ times per week & $\begin{array}{l}17 \\
(25.37)\end{array}$ & $9(20.93)$ & $8(33.33)$ & $9(27.27)$ & $8(23.53)$ & $6(31.58)$ & $\begin{array}{l}11 \\
(22.92)\end{array}$ & $9(22.5)$ & $8(29.63)$ & $8(25)$ & $\begin{array}{l}9 \\
(25.71)\end{array}$ \\
\hline \multicolumn{12}{|l|}{ Smoking, n (\%) } \\
\hline Yes & $3(4.48)$ & $2(4.65)$ & $1(4.17)$ & $2(6.06)$ & $1(2.94)$ & 0 & $3(6.25)$ & $2(5)$ & $1(3.7)$ & $2(6.25)$ & $1(2.86)$ \\
\hline No & $\begin{array}{l}64 \\
(95.52)\end{array}$ & $\begin{array}{l}41 \\
(95.35)\end{array}$ & $\begin{array}{l}23 \\
(95.83)\end{array}$ & $\begin{array}{l}31 \\
(93.94)\end{array}$ & $\begin{array}{l}33 \\
(97.06)\end{array}$ & $19(100)$ & $\begin{array}{l}45 \\
(93.75)\end{array}$ & $38(95)$ & $26(96.3)$ & $\begin{array}{l}30 \\
(93.75)\end{array}$ & $\begin{array}{l}34 \\
(97.14)\end{array}$ \\
\hline \multicolumn{12}{|l|}{ Alcohol, n (\%) } \\
\hline No alcohol & $9(13.43)$ & $9(20.93)$ & 0 & $5(15.15)$ & $4(11.76)$ & $3(15.79)$ & $6(12.5)$ & $8(20)$ & $1(3.7)$ & $6(18.75)$ & $3(8.57)$ \\
\hline$\leq 14$ unites per week & $\begin{array}{l}45 \\
(67.16)\end{array}$ & $\begin{array}{l}28 \\
(65.12)\end{array}$ & $\begin{array}{l}17 \\
(70.83)\end{array}$ & $\begin{array}{l}24 \\
(72.73)\end{array}$ & $\begin{array}{l}21 \\
(61.76)\end{array}$ & $\begin{array}{l}15 \\
(78.95)\end{array}$ & $30(62.5)$ & $26(65)$ & $\begin{array}{l}19 \\
(70.37)\end{array}$ & $\begin{array}{l}22 \\
(68.75)\end{array}$ & $\begin{array}{l}23 \\
(65.71)\end{array}$ \\
\hline$>14$ units per week & $13(19.4)$ & $6(13.95)$ & $7(29.17)$ & $4(12.12)$ & $9(26.47)$ & $1(5.26)$ & $12(25)$ & $6(15)$ & $7(25.93)$ & $4(12.5)$ & $\begin{array}{l}9 \\
(25.71)\end{array}$ \\
\hline Body mass index, mean (sd) & $\begin{array}{l}25.21 \\
(4.3)\end{array}$ & $\begin{array}{l}25.51 \\
(4.57)\end{array}$ & $\begin{array}{l}24.67 \\
(3.8)\end{array}$ & $\begin{array}{l}25.05 \\
(5.03)\end{array}$ & $\begin{array}{l}25.36 \\
(3.52)\end{array}$ & $\begin{array}{l}25.07 \\
(3.62)\end{array}$ & $\begin{array}{l}25.26 \\
(4.58)\end{array}$ & $\begin{array}{l}25.45 \\
(4.29)\end{array}$ & $\begin{array}{l}24.84 \\
(4.37)\end{array}$ & $\begin{array}{l}25.54 \\
(5.04)\end{array}$ & $\begin{array}{l}24.9 \\
(3.54)\end{array}$ \\
\hline \multicolumn{12}{|c|}{ Menstruation in the last year, $\mathrm{n}(\%)$} \\
\hline Yes & $\begin{array}{l}18 \\
(26.87)\end{array}$ & $\begin{array}{l}12 \\
(27.91)\end{array}$ & $6(25)$ & $\begin{array}{l}10 \\
(30.30)\end{array}$ & $8(23.53)$ & $7(36.84)$ & $\begin{array}{l}11 \\
(22.92)\end{array}$ & $11(27.5)$ & $7(25.93)$ & $8(25)$ & $\begin{array}{l}10 \\
(28.57)\end{array}$ \\
\hline No & $\begin{array}{l}49 \\
(73.13)\end{array}$ & $\begin{array}{l}31 \\
(72.09)\end{array}$ & $18(75)$ & $\begin{array}{l}23 \\
(69.70)\end{array}$ & $\begin{array}{l}26 \\
(76.47)\end{array}$ & $\begin{array}{l}12 \\
(63.16)\end{array}$ & $\begin{array}{l}37 \\
(77.08)\end{array}$ & $29(72.5)$ & $\begin{array}{l}20 \\
(74.07)\end{array}$ & $24(75)$ & $\begin{array}{l}25 \\
(71.43)\end{array}$ \\
\hline \multicolumn{12}{|l|}{ Number of births, $n$ (\%) } \\
\hline None & $4(5.97)$ & $2(4.65)$ & $2(8.33)$ & $1(3.03)$ & $3(8.82)$ & 0 & $4(8.33)$ & $2(5)$ & $2(7.41)$ & $2(6.25)$ & $2(5.71)$ \\
\hline One & $13(19.4)$ & $7(16.28)$ & $6(25)$ & $4(12.12)$ & $9(26.47)$ & $2(10.53)$ & 11 & $8(20)$ & $5(18.52)$ & $5(15.63)$ & 8 \\
\hline
\end{tabular}


Table 1 Baseline data stratified by experienced clinical reduction in the four symptom scales and overall composite scale (Continued)

\begin{tabular}{|c|c|c|c|c|c|c|c|c|c|c|c|}
\hline & \multirow[t]{2}{*}{ Total } & \multicolumn{2}{|c|}{$\begin{array}{l}\text { Hot flushes } \\
\text { Clinical reduction }\end{array}$} & \multicolumn{2}{|c|}{$\begin{array}{l}\text { Day-and-night } \\
\text { sweats } \\
\text { Clinical reduction }\end{array}$} & \multicolumn{2}{|c|}{$\begin{array}{l}\text { General sweating } \\
\text { Clinical reduction }\end{array}$} & \multicolumn{2}{|c|}{$\begin{array}{l}\text { Menopausal- } \\
\text { specific sleeping } \\
\text { problems } \\
\text { Clinical reduction }\end{array}$} & \multicolumn{2}{|c|}{$\begin{array}{l}\text { Overall } \\
\text { Clinical reduction }\end{array}$} \\
\hline & & Yes & No & Yes & No & Yes & No & Yes & No & Yes & No \\
\hline & & & & & & & $(22.92)$ & & & & (22.86) \\
\hline Two & $\begin{array}{l}36 \\
(53.73)\end{array}$ & $\begin{array}{l}24 \\
(55.81)\end{array}$ & $12(50)$ & $\begin{array}{l}19 \\
(57.58)\end{array}$ & $17(50)$ & $\begin{array}{l}14 \\
(73.68)\end{array}$ & $\begin{array}{l}22 \\
(45.83)\end{array}$ & $21(52.5)$ & $\begin{array}{l}15 \\
(55.56)\end{array}$ & $\begin{array}{l}18 \\
(56.25)\end{array}$ & $\begin{array}{l}18 \\
(51.43)\end{array}$ \\
\hline More than two & $14(20.9)$ & $\begin{array}{l}10 \\
(23.26)\end{array}$ & $4(16.67)$ & $9(27.27)$ & $5(14.71)$ & $3(15.79)$ & $\begin{array}{l}11 \\
(22.92)\end{array}$ & $9(22.5)$ & $5(18.52)$ & $7(21.88)$ & $7(20)$ \\
\hline \multicolumn{12}{|l|}{ Urinary Incontinence, n (\%) } \\
\hline No & $\begin{array}{l}23 \\
(34.33)\end{array}$ & $\begin{array}{l}17 \\
(39.53)\end{array}$ & $6(25)$ & $\begin{array}{l}15 \\
(45.45)\end{array}$ & $8(23.53)$ & $4(21.05)$ & $\begin{array}{l}19 \\
(39.58)\end{array}$ & $14(35)$ & $9(33.33)$ & $\begin{array}{l}14 \\
(43.75)\end{array}$ & $\begin{array}{l}9 \\
(25.71)\end{array}$ \\
\hline Yes & $\begin{array}{l}44 \\
(65.67)\end{array}$ & $\begin{array}{l}26 \\
(60.47)\end{array}$ & $18(75)$ & $\begin{array}{l}18 \\
(54.55)\end{array}$ & $\begin{array}{l}26 \\
(76.47)\end{array}$ & $\begin{array}{l}15 \\
(78.95)\end{array}$ & $\begin{array}{l}29 \\
(60.42)\end{array}$ & $26(65)$ & $\begin{array}{l}18 \\
(66.67)\end{array}$ & $\begin{array}{l}18 \\
(56.25)\end{array}$ & $\begin{array}{l}26 \\
(74.29)\end{array}$ \\
\hline \multicolumn{12}{|l|}{ Chronic disease, n (\%) } \\
\hline Yes & $13(19.4)$ & $8(18.6)$ & $5(20.83)$ & $7(21.21)$ & $6(17.65)$ & $4(21.05)$ & 9 (18.75) & $6(15)$ & $7(25.93)$ & $6(18.75)$ & $7(20)$ \\
\hline No & $54(80.6)$ & 35 (81.4) & $\begin{array}{l}19 \\
(79.17)\end{array}$ & $\begin{array}{l}26 \\
(78.79)\end{array}$ & $\begin{array}{l}28 \\
(82.35)\end{array}$ & $\begin{array}{l}15 \\
(78.95)\end{array}$ & $\begin{array}{l}39 \\
(81.25)\end{array}$ & $34(85)$ & $\begin{array}{l}20 \\
(74.07)\end{array}$ & $\begin{array}{l}26 \\
(81.25)\end{array}$ & $28(80)$ \\
\hline \multicolumn{12}{|c|}{ Current use of alternative treatment, $n(\%)$} \\
\hline Yes & $\begin{array}{l}50 \\
(74.63)\end{array}$ & $\begin{array}{l}31 \\
(72.09)\end{array}$ & $\begin{array}{l}19 \\
(79.17)\end{array}$ & $\begin{array}{l}23 \\
(69.70)\end{array}$ & $\begin{array}{l}27 \\
(79.41)\end{array}$ & $\begin{array}{l}16 \\
(84.21)\end{array}$ & $\begin{array}{l}34 \\
(70.83)\end{array}$ & $\begin{array}{l}26 \\
(65.00)\end{array}$ & $\begin{array}{l}24 \\
(88.89)\end{array}$ & $\begin{array}{l}23 \\
(71.88)\end{array}$ & $\begin{array}{l}27 \\
(77.14)\end{array}$ \\
\hline No & $\begin{array}{l}17 \\
(25.37)\end{array}$ & $\begin{array}{l}12 \\
(27.91)\end{array}$ & $5(20.83)$ & $\begin{array}{l}10 \\
(30.30)\end{array}$ & $7(20.59)$ & $3(15.79)$ & $\begin{array}{l}14 \\
(29.17)\end{array}$ & $\begin{array}{l}14 \\
(35.00)\end{array}$ & $3(11.11)$ & $9(28.13)$ & $\begin{array}{l}8 \\
(22.86)\end{array}$ \\
\hline $\begin{array}{l}\text { Duration of hot flushes, } \\
\text { mean (sd) }\end{array}$ & $\begin{array}{l}4.1 \\
(3.48)\end{array}$ & $\begin{array}{l}3.69 \\
(3.53)\end{array}$ & $\begin{array}{l}4.82 \\
(3.35)\end{array}$ & $\begin{array}{l}4.14 \\
(4.13)\end{array}$ & $\begin{array}{l}4.05 \\
(2.78)\end{array}$ & $\begin{array}{l}3.62 \\
(3.65)\end{array}$ & $\begin{array}{l}4.29 \\
(3.44)\end{array}$ & $\begin{array}{l}4.22 \\
(3.76)\end{array}$ & $\begin{array}{l}3.91 \\
(3.08)\end{array}$ & $\begin{array}{l}4.31 \\
(4.12)\end{array}$ & $\begin{array}{l}3.9 \\
(2.82)\end{array}$ \\
\hline \multicolumn{12}{|l|}{ Allocation status, n (\%) } \\
\hline Control & $\begin{array}{l}31 \\
(46.27)\end{array}$ & $\begin{array}{l}17 \\
(39.53)\end{array}$ & $\begin{array}{l}14 \\
(58.33)\end{array}$ & $\begin{array}{l}16 \\
(48.48)\end{array}$ & $\begin{array}{l}15 \\
(44.12)\end{array}$ & $9(47.37)$ & $\begin{array}{l}22 \\
(45.83)\end{array}$ & $16(40)$ & $\begin{array}{l}15 \\
(55.56)\end{array}$ & $\begin{array}{l}14 \\
(43.75)\end{array}$ & $\begin{array}{l}17 \\
(48.57)\end{array}$ \\
\hline Intervention & $\begin{array}{l}36 \\
(53.73)\end{array}$ & $\begin{array}{l}26 \\
(60.47)\end{array}$ & $\begin{array}{l}10 \\
(41.67)\end{array}$ & $\begin{array}{l}17 \\
(51.52)\end{array}$ & $\begin{array}{l}19 \\
(55.88)\end{array}$ & $\begin{array}{l}10 \\
(52.63)\end{array}$ & $\begin{array}{l}26 \\
(54.17)\end{array}$ & $24(60)$ & $\begin{array}{l}12 \\
(44.44)\end{array}$ & $\begin{array}{l}18 \\
(56.25)\end{array}$ & $\begin{array}{l}18 \\
(51.43)\end{array}$ \\
\hline
\end{tabular}

However, based on our study we found that the majority of women actually benefitted from the acupuncture treatment in one or more of the VMS dimensions we surveyed. Consequently, we suggest that the larger effect we find compared to previously reported effects may be due to our use of the rigorous validated outcome measure aimed to identify bothersome menopausal symptoms.

Our definition of a clinically relevant reduction was based on the data used for the validation of the MSQ. A clinically relevant reduction for the overall composite score was defined as a reduction of 6 points. This outcome, while not unidimensional, is potentially more sensitive. For detailed information on the MSQ please refer to the validation article [9].

The present study had a high participant adherence and used valid outcome measures with $100 \%$ response rate. Further, the intervention was found to be well-tolerated by the participants with only few mild side-effects and manageable for general practitioners. We used a brief and standardized acupuncture modality in order to evaluate the effectiveness and the determinants for a clinically relevant reduction in symptoms. Consequently, it may be transferred into most clinical settings, even outside of Denmark, due to the high external validity of the study. This standardized modality may be seen as a weakness for some, but was well accepted by the general practitioners who were doing the acupuncture [10].

The study was not powered to find clinical relevant reductions. However, significant results have $a<5 \%$ chance for a false positive result regardless of sample size. Due to the power in the present study some factors that may have clinically importance could not be detected. We excluded women who participated in other interventions for menopausal symptoms in order to minimize the influence of treatment effects of co-interventions, which makes the interpretation of the surveyed effects as caused by the acupuncture treatment more pertinent.

In this study we used WMA theory, in which acupuncture predominantly is understood by stimulating the 
Table 2 The effect of the three most important factors for each of the outcomes

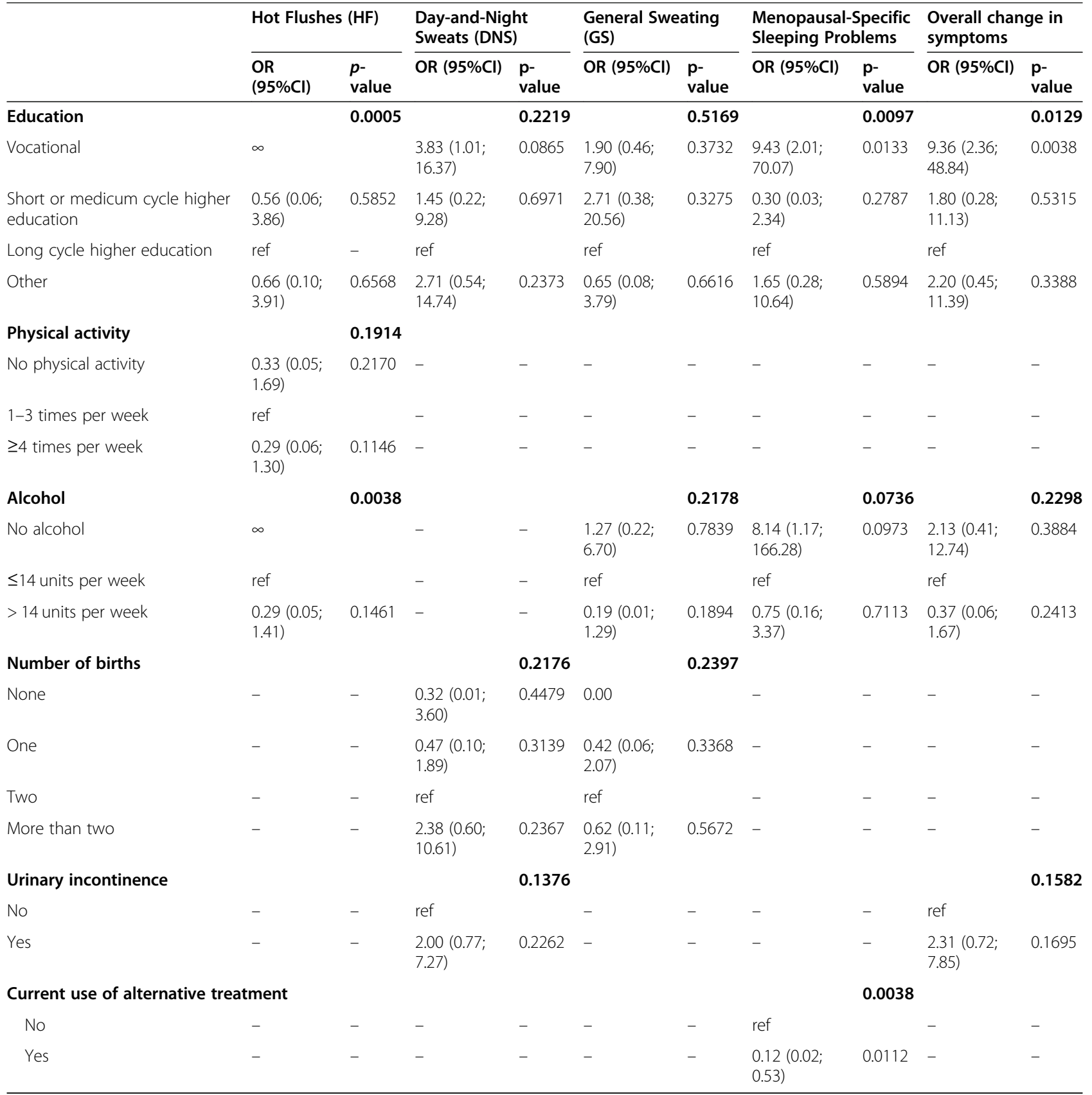

nervous system $[13,26]$. The ovaries and uterus are innervated by sympathetic nerves from the spinal cords segment Th11 to $\mathrm{S} 3$ and the parasympathetic nerves from the segment S2 to S4. Our acupuncture modality are all placed within these segments [26]. Furthermore, acupuncture has been shown to stimulate betaendorphins, serotonin and norepinephrine [26, 27], which are all believed to be related to temperature regulation and the generation of hot flushes [5]. However, the underlying mechanism of acupuncture still remains unknown, and it is likely that other acupuncture modalities also would find clinically relevant symptom reductions.

\section{Conclusions}

Women with vocational education were most likely to experience an improvement in their menopausal symptoms compared to women with higher education. Beyond level of education, other factors of some importance for clinically relevant reduction were no alcohol consumption, two or more births and urinary incontinence. The mechanisms are poorly understood and 
we recommend further research in order to qualify recommendations to clinicians.

\section{Abbreviations}

HF: Hot flushes; DNS: Day and night sweats; GS: General sweating; MSSP: Menopausal-specific sleeping problems; VSM: Vasomotor symptoms (VMS); WMA: Western medical acupuncture

\section{Acknowledgements}

We wish to acknowledge the GPs and the women who took part in this study, Palle Rosted, and Danish Society for Evidence Based Acupuncture for inspiration and counseling.

\section{Authors' contributions}

FBW, KSL, JB and VS contributed to the design and planning of the study. KSL conducted the study supported by FBW, JB and VS. Statistical analyses were carried out by CWB and VS. The manuscript was drafted by FBW but all authors revised the entire manuscript critically and approved the final version for publication.

\section{Funding}

The study was funded by the Idella Foundation, the University of Copenhagen, the Research Foundation of General Practice including the Foundation of Multipractice Studies. The funders had no role in the design of the study, the collection, analysis, and interpretation of data, or regarding decisions about publication.

\section{Availability of data and materials}

Anonymized data can be made available upon reasonable request.

\section{Ethics approval and consent to participate}

Approvals from the Committee on Health Research Ethics $\left(H^{-16016365}\right)$, the Committee of Multipractice Studies in General Practice (MPU 08-2016), and the Danish Data Protection Agency (SUND-2016-24) were obtained before enrolment. The study was conducted in accordance with the Declaration of Helsinki and Good Clinical Practice (ICH GCP). The trial was registered on ClinicalTrials.gov NCT02746497 before study start. All participants provided written consent.

\section{Consent for publication}

Not applicable. No personal information is included.

\section{Competing interests}

None declared.

\begin{abstract}
Author details
'The Research Unit for General Practice and Section of General Practice, Department of Public Health, University of Copenhagen, Copenhagen, Denmark. ${ }^{2}$ Department of Public Health, University of Southern Denmark, Research Unit of General Practice, Odense, Denmark. ${ }^{3}$ Primary Health Care Research Unit, Region Zealand, Sorø, Denmark.
\end{abstract}

Received: 7 July 2020 Accepted: 5 January 2021

Published online: 13 January 2021

\section{References}

1. Whiteley J, Wagner JS, Bushmakin A, Kopenhafer L, Dibonaventura M, Racketa J. Impact of the severity of vasomotor symptoms on health status, resource use, and productivity. Menopause (New York, NY). 2013;20(5):518-24.

2. Williams RE, Levine KB, Kalilani L, Lewis J, Clark RV. Menopause-specific questionnaire assessment in US population-based study shows negative impact on health-related quality of life. Maturitas. 2009;62(2):153-9.

3. Geukes M, van Aalst MP, Robroek SJ, Laven JS, Oosterhof H. The impact of menopause on work ability in women with severe menopausal symptoms. Maturitas. 2016;90:3-8.

4. Nelson HD. Menopause. Lancet (London, England). 2008;371:760-70

5. Stearns V, Ullmer L, Lopez JF, Smith Y, Isaacs C, Hayes D. Hot flushes. Lancet (London, England). 2002;360(9348):1851-61.

6. Avis NE, Crawford SL, Greendale G, Bromberger JT, Everson-Rose SA, Gold $E B$, et al. Duration of menopausal vasomotor symptoms over the menopause transition. JAMA Intern Med. 2015;175(4):531-9.
7. Col NF, Guthrie JR, Politi M, Dennerstein L. Duration of vasomotor symptoms in middle-aged women: a longitudinal study. Menopause (New York, NY). 2009;16(3):453-7.

8. Pachman DR, Jones JM, Loprinzi CL. Management of menopause-associated vasomotor symptoms: current treatment options, challenges and future directions. Int J Women's Health. 2010;2:123-35.

9. Lund KS, Siersma VD, Christensen KB, Waldorff FB, Brodersen J. Measuring bothersome menopausal symptoms: development and validation of the MenoScores questionnaire. Health Qual Life Outcomes. 2018;16(1):97.

10. Lund KS, Siersma V, Brodersen J, Waldorff FB. Efficacy of a standardised acupuncture approach for women with bothersome menopausal symptoms: a pragmatic randomised study in primary care (the ACOM study). BMJ Open. 2019;9(1):e023637.

11. Lund KS, Siersma V, Bang CW, Brodersen J, Waldorff FB. Sustained effects of a brief and standardised acupuncture approach on menopausal symptoms: post hoc analysis of the ACOM randomised controlled trial. Acupunct Med. 2020;964528420920280.

12. Avis NE, Coeytaux RR, Levine B, Isom S, Morgan T. Trajectories of response to acupuncture for menopausal vasomotor symptoms: the acupuncture in menopause study. Menopause. 2017;24(2):171-9.

13. White A. Western medical acupuncture: a definition. Acupuncture in medicine : journal of the British Medical Acupuncture Society. 2009;27(1):33-5.

14. Lund KS, Brodersen J, Siersma V, Waldorff FB. The efficacy of acupuncture on menopausal symptoms (ACOM study): protocol for a randomised study. Danish medical journal. 2017;64(3).

15. Johnson JWLJ. History and use of relative importance indices in organizational research. Organ Res Methods. 2004;7:238-57.

16. Ekholm O JH, Davidsen M, Christensen Al. Alternativ behandling. Sundhedsog sygelighedsundersøgelsen 2017. Copenhagen: Statens Institut for Folkesundhed, SDU.

17. Vase L, Baram S, Takakura N, Yajima H, Takayama M, Kaptchuk TJ, et al. Specifying the nonspecific components of acupuncture analgesia. Pain. 2013;154(9):1659-67.

18. Torgerson DJ, Thomas RE, Campbell MK, Reid DM. Alcohol consumption and age of maternal menopause are associated with menopause onset Maturitas. 1997;26(1):21-5.

19. Gavaler JS, Imhoff AF, Pohl CR, Rosenblum ER, Van Thiel DH. Alcoholic beverages: a source of estrogenic susbstances? Alcohol Alcoholism. 1987;1: 545-9.

20. Avis NE, Legault C, Coeytaux RR, Pian-Smith M, Shifren JL, Chen W, et al. A randomized, controlled pilot study of acupuncture treatment for menopausal hot flashes. Menopause. 2008;15(6):1070-8.

21. Borud EK, Alraek T, White A, Fonnebo V, Grimsgaard S. The effect of TCM acupuncture on hot flushes among menopausal women (ACUFLASH) study: a study protocol of an ongoing multi-Centre randomised controlled clinical trial. BMC Complement Altern Med. 2007;7:6.

22. Kim KH, Kang KW, Kim DI, Kim HJ, Yoon HM, Lee JM, et al. Effects of acupuncture on hot flashes in perimenopausal and postmenopausal women-a multicenter randomized clinical trial. Menopause. 2010;17(2):269-80.

23. Nedeljkovic M, Tian L, Ji P, Deglon-Fischer A, Stute P, Ocon E, et al. Effects of acupuncture and Chinese herbal medicine (Zhi mu 14) on hot flushes and quality of life in postmenopausal women: results of a four-arm randomized controlled pilot trial. Menopause. 2014;21 (1):15-24.

24. Painovich JM, Shufelt $C L$, Azziz R, Yang $Y$, Goodarzi MO, Braunstein GD, et al. A pilot randomized, single-blind, placebo-controlled trial of traditional acupuncture for vasomotor symptoms and mechanistic pathways of menopause. Menopause. 2012;19(1):54-61.

25. Vincent A, Barton DL, Mandrekar JN, Cha SS, Zais T, Wahner-Roedler DL, et al. Acupuncture for hot flashes: a randomized, sham-controlled clinical study. Menopause. 2007;14(1):45-52.

26. Rosted P Akupunktur: på naturvidenskabeligt grundlag. 1th ed. Århus: Klim; 2003.

27. Ee C, French SD, Xue CC, Pirotta M, Teede H. Acupuncture for menopausal hot flashes: clinical evidence update and its relevance to decision making. Menopause (New York, NY). 2017;24(8):980-7.

\section{Publisher's Note}

Springer Nature remains neutral with regard to jurisdictional claims in published maps and institutional affiliations. 